\title{
An empirical test of the independence between declarative and procedural working memory in Oberauer's (2009) theory
}

\author{
Pierre Barrouillet • Lucie Corbin • Isabelle Dagry • \\ Valérie Camos
}

Published online: 13 December 2014

(C) Psychonomic Society, Inc. 2014

\begin{abstract}
It has recently been suggested that working memory could be conceived as two symmetrical subsystems with analogous structure and processing principles: a declarative working memory storing objects of thought available for cognitive operations, and a procedural working memory holding representations of what to do with these objects (Oberauer, Psychology of learning and motivation 51: 45-100, 2009). Within this theoretical framework, the two subsystems are thought to be independent and fueled by their own capacity. The present study tested this hypothesis through two experiments using a complex span task in which participants were asked to maintain consonants for further recall while performing response selection tasks. In line with Oberauer's conception, the load of the procedural working memory was varied by manipulating the number of stimulus-response mappings of the response selection task. Increasing the number of these mappings had a strong detrimental effect on recall performance. Besides contradicting Oberauer's proposal, this finding supports models that assume a resource-sharing between processing and storage in working memory.
\end{abstract}

\section{Keywords Working memory $\cdot$ Memory}

P. Barrouillet $\cdot$ I. Dagry

Department of Psychology, University of Geneva, Geneva,

Switzerland

L. Corbin

LEAD - CNRS, Université de Bourgogne, Dijon, France

V. Camos

Department of Psychology, University of Fribourg, Fribourg, Switzerland

P. Barrouillet $(\square)$

Faculté de Psychologie et des Sciences de l'Education, Université de Genève, 40 bd du Pont d'Arve, 1211 Genève 4, Switzerland

e-mail: pierre.barrouillet@unige.ch
Working memory is a system devoted to the temporary storage of information in view of its processing. Although the first theories regarded processing and storage as supported by distinct mechanisms and structures (Baddeley, 1986), further theories assumed that the two functions compete for a common resource usually referred to as an attentional or executive capacity (Anderson, Reder, \& Lebière, 1996; Barrouillet, Bernardin, \& Camos, 2004; Case, 1985; Just \& Carpenter, 1992). This latter conception is reflected in the structure of the complex span tasks designed to assess working memory capacity, in which the memorization of items is hindered by a concurrent processing. The hypothesis that processing and storage share a common resource is usually evidenced by the fact that any increase in processing demand results in poorer recall performance, a trade-off that has been extensively evidenced (e.g., Anderson et al., 1996; Barrouillet et al., 2004; Barrouillet, Portrat, \& Camos, 2011; Case et al. 1982).

However, the independence hypothesis has been renewed by recent theorizing. Oberauer $(2009,2010)$ has suggested distinguishing between a declarative and a procedural working memory. The former, constituting the storage part of working memory, would make representations available for processing, whereas the latter would be devoted to the representation and selection of the cognitive operations. The two systems would have the same tri-partite structure corresponding to three levels of selection of representation. Within the declarative part, a first level is constituted by elements in longterm memory activated above baseline by perceptual input or spreading activation. At a second level, a small number of these elements are integrated into a region of direct access that makes them immediately accessible for treatment. Finally, a focus of attention can select one element from this region as the object of a cognitive operation. Representations in the procedural part are procedures stored in a procedural longterm memory. At the lowest level, a subset of these procedures would be activated at any time by representations of goals or 
by stimuli to which they have been applied in the past. A more central component of the procedural system, called the bridge, holds the currently operative task set that is in control of thought and action. This task set consists of a set of stimulus-response mappings that specifies the cognitive operations to be undertaken for a limited set of conditions as well as the results that can be expected. The bridge can be considered as the procedural counterpart of the region of direct access. In the same way as the region of direct access holds temporary bindings between representations, the bridge can hold bindings that are not limited to the learned associations in long-term memory, such as the arbitrary stimulus-response mappings implemented in experimental instructions. It is worth noting that these arbitrary bindings can be quickly and flexibly set up, constituting "prepared reflexes" (Hommel, 1998) in such a way that stimuli activate their corresponding response automatically.

One of the main assumptions of this model is that analogous mechanisms serve to select declarative and procedural representations, a prediction supported by empirical findings (Souza, Oberauer, Gade, \& Druey, 2012). Another strong assumption is that the region of direct access and the bridge are independent systems with separate capacities, in such a way that "increasing the load of declarative working memory should not affect the efficiency of executing a task held in procedural working memory, and conversely, increasing the load on procedural working memory should not impair retention of information in declarative memory" (Oberauer, 2009, p. 74). According to Oberauer, "an informative test of the above prediction would have to involve a well-practiced task for the manipulation of procedural complexity, which no longer relies on declarative instructions. Alternatively, a nonverbal WM load that does not interfere with verbal instructions held in declarative WM could be used to manipulate declarative WM load. As far as I am aware, no such study has yet been conducted" (Oberauer, 2009, p. 74).

It is this prediction that the present study aimed at testing. We used a complex span task paradigm in which participants were asked to maintain series of letters for recall, while performing a concurrent response selection task after each letter presentation. Oberauer describes procedural representations "as condition-action rules, with the condition describing the circumstances to which the procedure applies, and the action component describing what is to be done" (Oberauer, 2010, p. 279). From this definition, we assumed that the load on what he calls procedural working memory could be increased by increasing the number of stimulus-response mappings (either two or four) that the response selection task involved. The hypothesis of independence would predict no effect of this manipulation on recall performance. By contrast, the resource-sharing hypothesis predicts lower recall performance with more complex task set. Indeed, increasing the number of choice alternatives in a response selection task increases the time needed to select the appropriate response, a phenomenon referred to as the law of Hick and Hyman (Hick, 1952; Hyman, 1953) and typically attributed to increased response selection difficulty. It has been shown that increasing response selection difficulty places higher demand on attention and executive control (Szmalec et al. 2005), which is the resource assumed to be shared between processing and storage by the resource-sharing theories evoked above.

It should be noted that Oberauer (2009) points out that his prediction of independence is complicated by the fact that task sets are units of procedural knowledge, but experimental instructions correspond to declarative knowledge. For novel and not yet practiced tasks, participants could hold instructions in declarative working memory. Thus, increasing task set complexity could affect the concurrent retention of information in declarative working memory due to interference between instructions and memory items if participants maintain these instructions in a declarative format. To avoid this drawback, we used stimulus-response mappings that are difficult to verbalize. Participants were instructed to press keys in response to either two or four Chinese ideograms (Fig. 1). Moreover, each stimulus-response mapping was trained to set up the "prepared reflexes" that would no longer require the use of declarative representation of instructions for their implementation. Cohen-Kdoshay and Meiran (2007) have demonstrated that, in experimental settings, stimuli can automatically activate their associated response even after little practice. Thus, we assumed that the combination of new meaningless stimuli difficult to encode verbally and a training session strengthening the stimulus-response mappings would reduce the probability of a declarative representation of instructions that could interfere with the memoranda.

\section{Experiment 1}

\section{Participants}

Twenty-six students (mean age $=19.5$ years, $S D=1.1$; two males) at the Université de Bourgogne received course credit to participate.

\section{Materials and procedure}

Participants performed a computer-paced complex span task in which they had to memorize series of six consonants, each consonant being followed by four ideograms. We selected six familiar Chinese ideograms for their simple and very distinctive characteristics. Each trial began by a ready signal (an asterisk) centered on screen for $1000 \mathrm{~ms}$ followed by the first consonant presented for $1000 \mathrm{~ms}$. This letter was immediately followed by four ideograms presented successively on screen 
Fig. 1 Ideograms used in the experiments with their mappings on an AZERTY keyboard

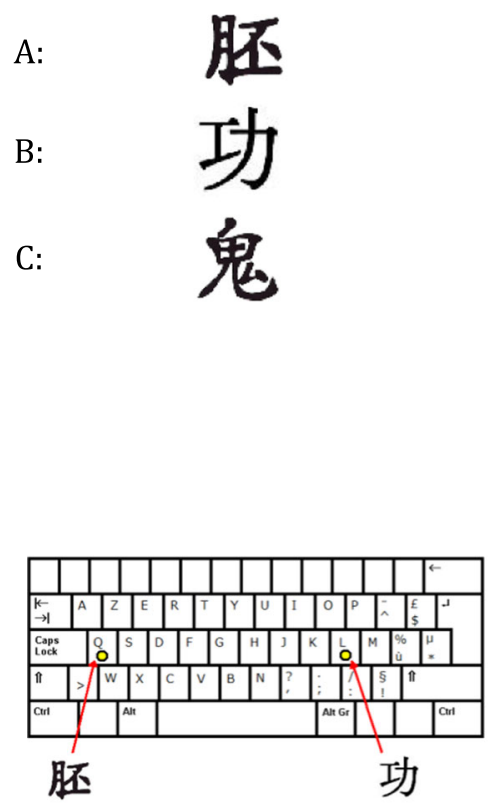

2-ideogram condition
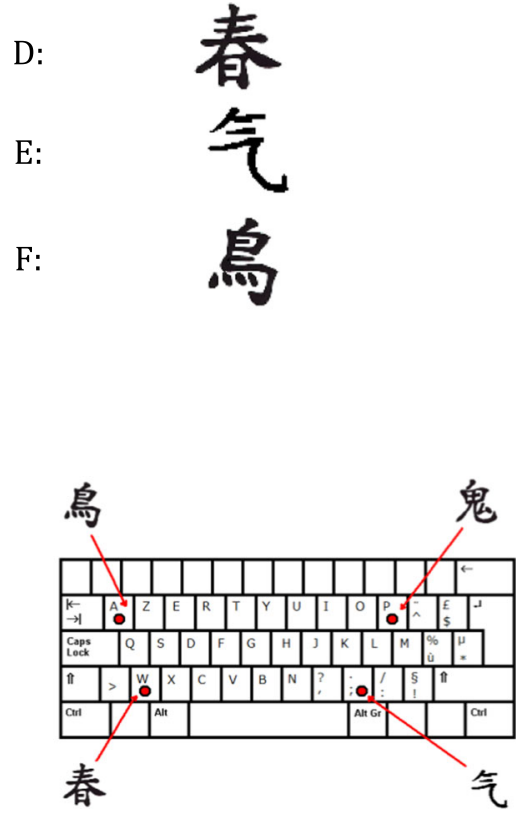

4-ideogram condition at a rate of one ideogram per second ( $800 \mathrm{~ms}$ on and $200 \mathrm{~ms}$ off). Consonants were randomly selected for each trial and each participant, as well as the ideograms from two different pools depending on the condition (ideograms $\mathrm{A}$ and $\mathrm{B}$ in Fig. 1 in the two-ideogram condition and ideograms C, D, $\mathrm{E}$, and $\mathrm{F}$ in the four-ideogram condition). There were two blocks of eight trials per condition. These four blocks were presented in two different orders $(2-4-2-4$ and 4-2-4-2 ideograms) counterbalanced across participants.

Participants read the letters aloud and pressed the key corresponding to the ideogram appearing on screen as quickly as possible (ideograms A, B, C, D, E, and F were associated on an AZERTY keyboard with keys $q, l, p, w, ;$ and $a$ respectively). They were informed about the nature of the forthcoming block and instructed to leave their forefingers on the corresponding keys in the two-ideogram condition, and their forefingers and middle fingers in the four-ideogram condition. When the fourth ideogram was presented, the next consonant appeared, and so on. At the end of the trial, the word "Rappel" (recall) was displayed on screen. Participants wrote down the remembered letters in correct order by filling out six box-frames while leaving blank the boxes corresponding to forgotten letters.

This experimental session was preceded by a training session on the ideogram task. Participants were invited to familiarize themselves first with the stimulus-response mappings by performing the ideogram task with two blocks of 20 ideograms, one per experimental condition (i.e., two- and four-ideogram conditions), presented in the same order and at the same rate as in the forthcoming experimental session. Feedback was provided during this training with a beep sounding after each incorrect response. This training session was repeated until the participant reached a criterion of $80 \%$ correct responses on the 40 ideograms presented. After this training on the ideogram task, participants performed two trials of the complex span task per condition that were repeated until the same $80 \%$ correct criterion in the ideogram task was reached.

\section{Results and discussion}

Concerning the training phase, participants completed on average 2.16 times $(S D=0.69)$ the training on the ideogram task before reaching the criterion of $80 \%$ correct. On the last completion of the training, the two- and four-ideogram conditions elicited $96 \%$ and $82 \%$ of correct responses, respectively. The data from two participants who did not reach the 80 $\%$ criterion during the complex span task training were discarded.

As far as the experimental complex span task was concerned, we first verified that the order of presentation of the two experimental conditions (i.e., two vs. four ideograms) had no effect on any of the dependent variables and was not involved in any significant interaction. Consequently, order was removed from the analyses. Concerning the ideogram task, the two-ideogram condition elicited a higher rate of correct responses than the four-ideogram condition (92\%, $S D=4$, and $84 \%, S D=8$, respectively), $F(1,23)=32.26$, $p<.001, \eta_{\mathrm{p}}^{2}=.58$, and shorter response times (433 ms, $S D=$ 38 , and $544 \mathrm{~ms}, S D=27$, respectively), $F(1,23)=303.19, p<$ $.001, \eta_{\mathrm{p}}^{2}=.93$. As the resource-sharing hypothesis predicted, recall performance was better in the two- than in the four- 
ideogram condition, with $70 \%(S D=14)$ and $61 \%(S D=11)$ of letters recalled in correct position, respectively, $F(1,23)=$ $11.09, p<.01, \eta_{\mathrm{p}}^{2}=.33, d=.69$. Because there was a strong difference between the two experimental conditions in the rate of correct responses on the ideogram task, all the trials in which participants did not reach $80 \%$ of correct responses were removed from the analysis. This trimming procedure led to including an average of $15.0(S D=1.5)$ and $10.7(S D=4.6)$ out of 16 trials in the two- and four-ideogram conditions, respectively. The difference in the rate of correct responses in the two- and four-ideogram conditions was reduced ( $94 \%$, $S D=3$, and $90 \%, S D=3$, respectively), but still significant, $F$ $(1,23)=33.76, p<.001, \eta_{\mathrm{p}}^{2}=.60$. It disappeared when considering rates corrected for guessing ${ }^{1}(88 \%, S D=6$, and $87 \%, S D=4$, respectively), $F<1, \eta_{\mathrm{p}}^{2}=.02$, while mean response times remained unchanged (433 $\mathrm{ms}, S D=37$, and $544 \mathrm{~ms}, S D=28$, respectively), $F(1,23)=303.11, p<.001$, $\eta_{\mathrm{p}}^{2}=.93$. Interestingly, this trimming procedure did not affect the results concerning recall performance that was still significantly better in the two- than in the four-ideogram condition $(71 \%, S D=15$, and $63 \%, S D=12$, respectively), $F(1,23)=$ $8.12, p<.01, \eta_{\mathrm{p}}^{2}=.26, d=.58$.

This first attempt to test the independence between procedural and declarative WM showed that increasing the load on procedural working memory had a detrimental effect on the amount of declarative knowledge concurrently maintained. However, this result could have been due to some characteristic of our procedure. Indeed, the training program included 20 trials for both conditions. However, because there were twice the number of ideograms in the four- than in the twoideogram condition, each of them benefited from two times fewer exposures. This could have resulted in a lower level of automation and some more frequent recourse to a declarative representation of the stimulus-response mappings that could have disrupted memory performance. Thus, we replicated the experiment using a more balanced training program. In addition, participants were instructed to perform the task under articulatory suppression to avoid any verbal coding of the ideograms and their association with the corresponding key.

\section{Experiment 2}

\section{Participants}

Thirty-six students (mean age $=20.8$ years, $S D=3.1,2$ males) at the Universite de Genève received course credit to participate.

Materials and procedure

Materials and procedure were the same as in Experiment 1 with three exceptions. First, participants performed the training and the experimental session under articulatory suppression. Second, the additional difficulty incurred by concurrent articulation led us to reduce the length of the series of letters to be recalled to five instead of six as in Experiment 1. Third, the training schedule involved four steps. First, participants were familiarized with the ideogram task with feedback as in Experiment 1, except that each training round included a block of 20 trials in the two-ideogram condition, and a block of 40 trials in the four-ideogram condition in such a way that each ideogram was presented ten times. An $80 \%$ criterion was requested for both conditions, the entire training being repeated until participants reached this criterion in both blocks. In the second step, participants practiced the articulatory task, repeating the syllable $b a$ over $20 \mathrm{~s}$ at a fixed pace of 86 beats per minute given by a metronome. The third step consisted of the combination of the ideogram and the articulatory tasks, participants responding to 20 ideograms in each of the two conditions while repeating the syllable $b a$ at the rhythm of the metronome. In the last step, participants were familiarized with the complex span task with two series in each experimental condition.

The complex span task was the same as in Experiment 1 except that participants performed the task under articulatory suppression. The rhythm of the metronome ( 86 beats per minute) constituted an auditory background throughout the task. For each trial, participants were instructed to begin articulating $b a$ immediately after having read the first consonant, to interrupt this articulation only for reading the other consonants, and to stop it when the word "Rappel" (recall) was displayed on screen.

\section{Results and discussion}

Seven participants who had not yet reached the criterion after their sixth attempt in the first step of the training program were dismissed from the study. The remaining 29 participants reached $96 \%$ and $89 \%$ of correct responses in the two- and four-ideogram conditions, respectively, on the last completion of this first training step and were included in the experimental session.

As in Experiment 1, the order of presentation of the two experimental conditions had no effect and was consequently not included in the following analyses. With regard to the ideogram task, the rate of correct responses was slightly lower than in the previous experiment, with $88 \%(S D=7)$ and $77 \%$ $(S D=10)$ for the two- and four-ideogram conditions respectively, $F(1,28)=64.20, p<.001, \eta_{\mathrm{p}}^{2}=.70$. The twoideogram condition still elicited shorter response time (RTs) than the four-ideogram condition ( $447 \mathrm{~ms}, S D=51$, and 542 $\mathrm{ms}, S D=30$, respectively), $F(1,28)=277.11, p<.001, \eta_{\mathrm{p}}^{2}=$ .91. As in Experiment 1 and in line with our prediction, the percentage of letters correctly recalled was higher in the twothan in the four-ideogram condition $(61 \%, S D=14$, and 53 
$\%, S D=14$, respectively), $F(1,28)=9.70, p<.01, \eta_{\mathrm{p}}^{2}=.26$, $d=.58$. As we had done in the previous experiment, a further analysis was conducted by removing all the trials in which participants did not reach $80 \%$ of correct responses on the ideogram task. This trimming procedure led us to discard two participants who had no remaining trials in the four-ideogram condition. The mean number of trials analyzed in the 27 remaining participants was $14.0(S D=3.3)$ and $9.3(S D=$ $4.7)$ in the two- and four-ideogram conditions, respectively. In these trials, the response selection task elicited $91 \%(S D=4)$ and $86 \%(S D=3)$ of correct responses for the two- and fourideogram conditions, respectively, $F(1,26)=66.69, p<.001$, $\eta_{\mathrm{p}}^{2}=.72$, with mean RTs of $448 \mathrm{~ms}(S D=53)$ and $545 \mathrm{~ms}(S D$ $=34)$, respectively, $F(1,26)=262.30, p<.001, \eta_{\mathrm{p}}^{2}=.91$. As in Experiment 1, the rates of correct responses no longer differed between conditions when considering rates corrected for guessing, $F<1$. Interestingly, the effect of the number of stimulus-response mappings on recall performance was even clearer with $64 \%(S D=15)$ and $54 \%(S D=15)$ of letters correctly recalled in the two- and four-ideogram conditions, respectively, $F(1,26)=13.90, p<.001, \eta_{\mathrm{p}}^{2}=.35, d=.72$. In summary, a more balanced training schedule and the addition of an articulatory suppression throughout the ideogram task did not modify the results observed in the first experiment.

\section{General discussion}

In two experiments, we observed that, all other things being equal, varying the number of stimulus-response mappings in a response selection task disrupts concurrent maintenance of verbal information. Provided that increasing the number of stimulus response-mappings results in an increased load on procedural working memory as conceived by Oberauer (2009, 2010), these findings contradict his hypothesis of independence between declarative and procedural working memory. They are also at odds with Gade, Druey, Souza, and Oberauer (2014) who recently reaffirmed the independence hypothesis and tested it using a different paradigm to our own. Four experiments did not reveal any clear effect of increased procedural load on declarative working memory. However, the absence of temporal constraint in their tasks (cf. Oberauer \& Lewandowsky, 2013) and the small declarative memory load they used (maximum of four items) could explain the failure to reveal the interaction we observed between the two systems.

It is worth noting that the present results do not provide evidence for or against the existence of two separable entities like procedural and declarative working memories or the hypothesis that these entities store representations selected through analogous mechanisms. Some theories would reject the idea that condition-action rules are knowledge that can be represented in working memory (e.g., Anderson, 1993), whereas others assume that cognitive operations share a common mental space with the representations on which they operate (Case, 1985). In the same way, the present findings remain neutral about the specific mechanism through which a more demanding processing impairs concurrent maintenance. Models that assume a continuous sharing of resources between processing and storage would invoke the fact that a more difficult response selection task consumes more attentional resources that are no longer available for storage (e.g., Anderson et al., 1996; Case, 1985; Just \& Carpenter, 1992), whereas models positing a time-based instead of a continuous sharing could invoke the longer processing times involved by more difficult response selections (e.g., Barrouillet et al., 2004).

Whatever the precise mechanism through which the tradeoff occurs, our results echo several previous findings suggesting a strong dependence between the processing and storage functions of working memory (see Barrouillet et al., 2011, for review). Nonetheless, they are novel in that we are not aware of a study having manipulated the demand of the processing component of complex span tasks by varying the number of stimulus-response mappings this processing component involves. The fact that the ideogram task relies on visual stimuli and motor responses whereas the storage component of our task was verbal in nature reinforces the hypothesis that processing and storage functions share a domain-general and central resource. Indeed, the observed trade-off between processing and storage does not seem to be due to some maintenance of task instructions in a declarative format. The requirement of performing the task under articulatory suppression left the results unchanged. In the same way, van't Wout et al., (2013) found that phonological representations are not used to activate or maintain stimulus-response mappings. Moreover, the training programs proved effective in setting up the "prepared reflexes" evoked by Hommel (1998), as the analysis of RTs suggests. The mean RTs in the ideogram task were comparable to those observed in tasks requiring overlearned judgments such as parity or magnitude judgments on one-digit numbers (Liefooghe, Barrouillet, Vandierendonck, \& Camos, 2008).

In summary, manipulating the procedural complexity of a well-practiced task as Oberauer (2009) suggested has a detrimental effect on concurrent maintenance of verbal information. This result suggests that conceptualizing the storage and processing functions of working memory as supported respectively by a declarative and a procedural working memory that have separate capacities does not seem to be a promising theoretical framework.

\section{Footnotes}

1. Rates corrected for guessing were calculated using the formula 
$P_{c o r}=\left(P_{o b s}-P_{g}\right) / 1-P g$

where $P_{\text {cor }}$ corresponds to the corrected probability of correct response, $P_{o b s}$ to the observed probability, and $P_{g}$ to the probability to give a correct response by guessing, which was .50 in the two-ideogram condition and .25 in the fourideogram condition.

Acknowledgments We thank Pascal Morgan for his help in collecting data. This research was supported by a grant from the Agence Nationale de la Recherche $\mathrm{N}^{\circ}$ ANR-08-BLAN-045 to Valérie Camos.

\section{References}

Anderson, J. R. (1993). Rules of the mind. Hillsdale, NJ: Lawrence Erlbaum Associates.

Anderson, J. R., Reder, L. M., \& Lebière, C. (1996). Working memory: Activation limitations on retrieval. Cognitive Psychology, 30, 221256.

Baddeley, A. D. (1986). Working memory. Oxford: Clarendon Press.

Barrouillet, P., Bernardin, S., \& Camos, V. (2004). Time constraints and resource sharing in adults' working memory spans. Journal of Experimental Psychology: General, 133, 83-100.

Barrouillet, P., Portrat, S., \& Camos, V. (2011). On the law relating processing to storage in working memory. Psychological Review, 118, 175-192.

Case, R. (1985). Intellectual development: Birth to adulthood. New York: Academic Press.

Case, R., Kurland, M. D., \& Goldberg, J. (1982). Operational efficiency and the growth of short-term memory span. Journal of Experimental Child Psychology, 33, 386-404.

Cohen-Kdoshay, O., \& Meiran, N. (2007). The representation of instructions in working memory leads to autonomous response activation:
Evidence from the first trials in the flanker paradigm. Quarterly Journal of Experimental Psychology, 60, 1140-1154.

Gade, M., Druey, M. D., Souza, A. S., \& Oberauer, K. (2014). Interference within and between declarative and procedural representations in working memory. Journal of Memory and Language, 76, 174-194.

Hick, W. (1952). On the rate of gain of information. Quarterly Journal of Experimental Psychology, 4, 11-36.

Hommel, B. (1998). Automatic stimulus-response translation in dual task performance. Journal of Experimental Psychology: Human Perception and Performance, 24, 1368-1384.

Hyman, R. (1953). Stimulus information as a determinant of reaction time. Journal of Experimental Psychology, 45, 188-196.

Just, M. A., \& Carpenter, P. A. (1992). A capacity theory of comprehension: Individual differences in working memory. Psychological Review, 99, 122-149.

Liefooghe, B., Barrouillet, P., Vandierendonck, A., \& Camos, V. (2008). Working memory costs of task switching. Journal of Experimental Psychology: Learning, Memory, and Cognition, 34, 478-494.

Oberauer, K. (2009). Design for a working memory. In B. H. Ross (Ed.), Psychology of learning and motivation (Vol. 51, pp. 45-100). San Diego, CA: Elsevier.

Oberauer, K. (2010). Declarative and procedural working memory: common principles, common capacity limits? Psychologica Belgica, 50, 277-308.

Oberauer, K., \& Lewandowsky, S. (2013). Evidence against decay in verbal working memory. Journal of Experimental Psychology: General, 142, 380-411.

Souza, A. S., Oberauer, K., Gade, M., \& Druey, M. D. (2012). Processing of representations in declarative and procedural working memory. Quarterly Journal of Experimental Psychology, 65, 1006-1033.

Szmalec, A., Vandierendonck, A., \& Kemps, E. (2005). Response selection involves executive control: Evidence from the selective interference paradigm. Memory \& Cognition, 33, 531-541.

van't Wout, F., Lavric, A., \& Monsell, S. (2013). Are stimulus-response rules represented phonologically for task-set preparation and maintenance? Journal of Experimental Psychology: Learning, Memory, and Cognition, 39, 1538-1551. 